\title{
NGNP Risk Management Database: A Model for Managing Risk
}

September 2009

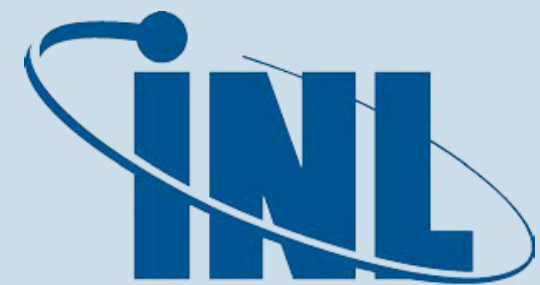

Idaho National Laboratory

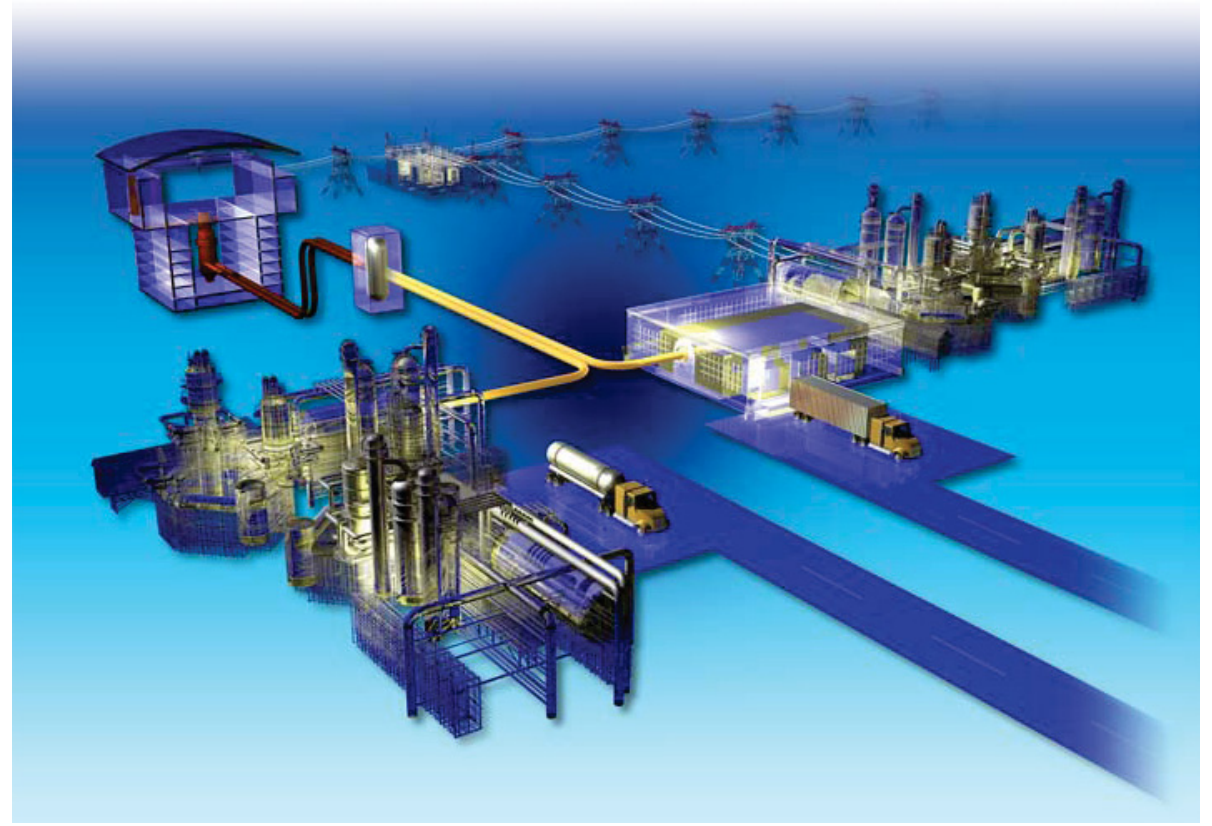

The INL is a U.S. Department of Energy National Laboratory operated by Battelle Energy Alliance. 


\section{DISCLAIMER}

This information was prepared as an account of work sponsored by an agency of the U.S. Government. Neither the U.S. Government nor any agency thereof, nor any of their employees, makes any warranty, expressed or implied, or assumes any legal liability or responsibility for the accuracy, completeness, or usefulness, of any information, apparatus, product, or process disclosed, or represents that its use would not infringe privately owned rights. References herein to any specific commercial product, process, or service by trade name, trade mark, manufacturer, or otherwise, does not necessarily constitute or imply its endorsement, recommendation, or favoring by the U.S. Government or any agency thereof. The views and opinions of authors expressed herein do not necessarily state or reflect those of the U.S. Government or any agency thereof. 


\section{NGNP Risk Management Database: A Model for Managing Risk}

September 2009

Idaho National Laboratory

Next Generation Nuclear Plant Project

Idaho Falls, Idaho 83415

http://www.inl.gov

Prepared for the

U.S. Department of Energy

Office of Nuclear Energy

Under DOE Idaho Operations Office

Contract DE-AC07-05ID14517 
This page intentionally left blank. 


\section{Next Generation Nuclear Plant (NGNP) Project}

\section{NGNP Risk Management Database: \\ A Model for Managing Risk}

INL/EXT-09-16778

September 2009
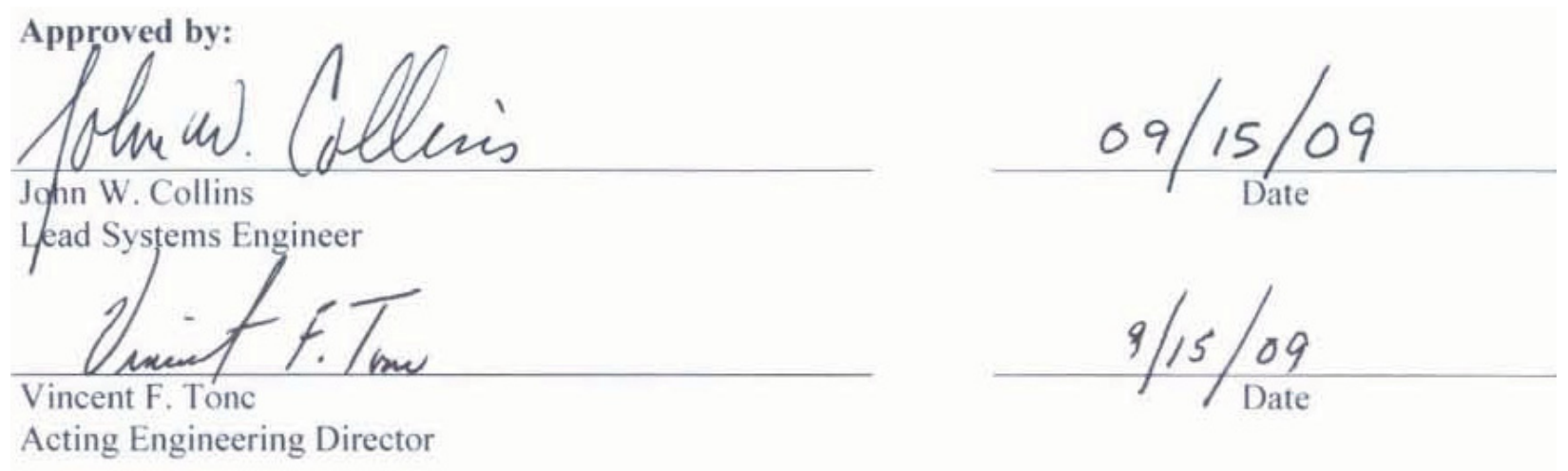
This page intentionally left blank. 


\section{REVISION LOG}

\begin{tabular}{|l|l|l|l|}
\hline Rev. & Date & Affected Pages & Revision Description \\
\hline & & & \\
\hline & & & \\
\hline & & & \\
\hline & & & \\
\hline & & & \\
\hline & & & \\
\hline & & & \\
\hline & & & \\
\hline & & & \\
\hline & & & \\
\hline & & & \\
\hline
\end{tabular}


This page intentionally left blank. 


\section{CONTENTS}

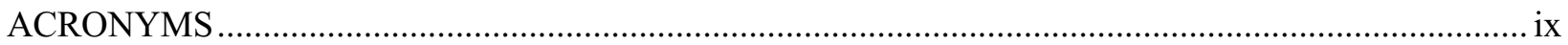

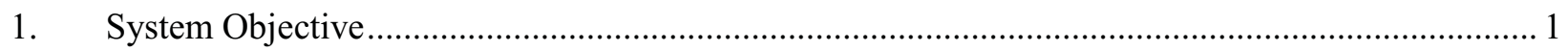

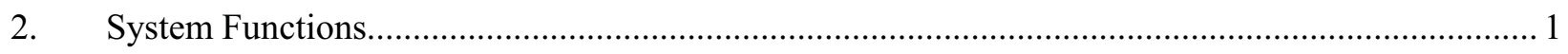

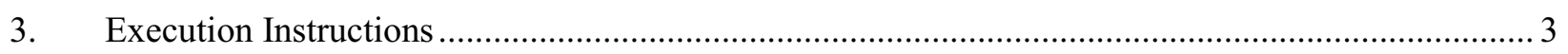

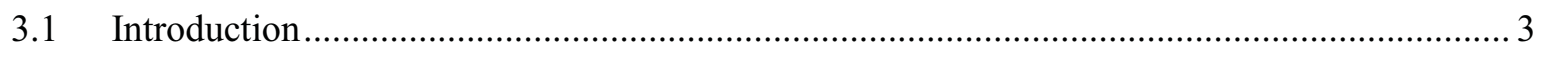

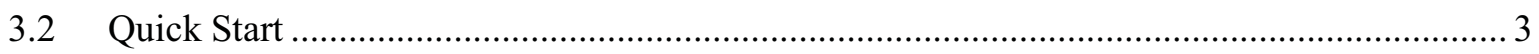

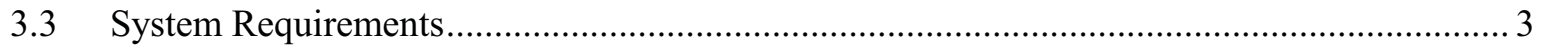

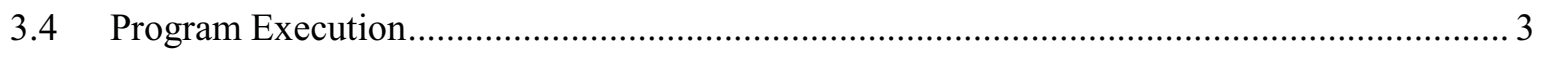

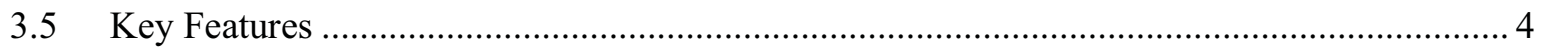

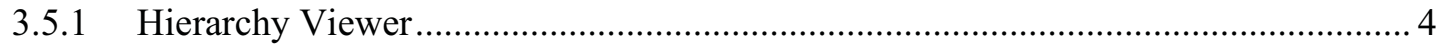

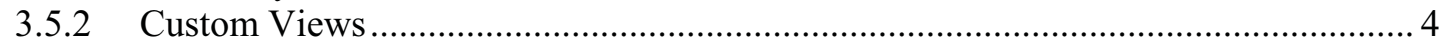

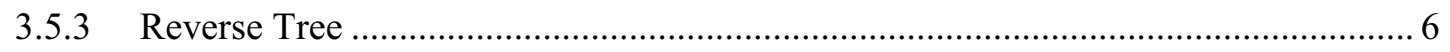

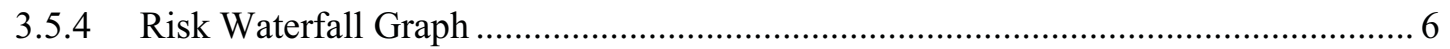

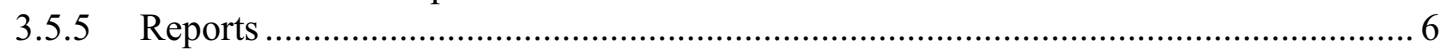

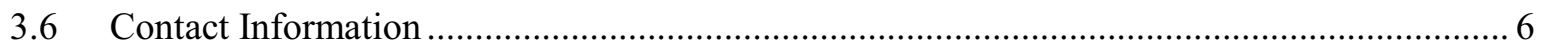

\section{FIGURES}

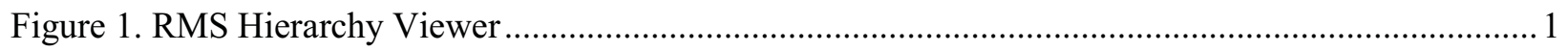

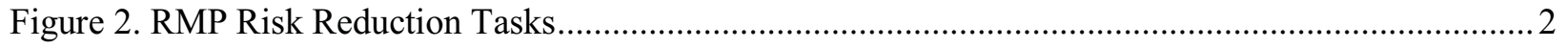

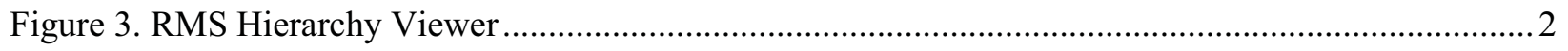

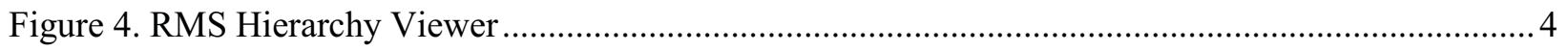

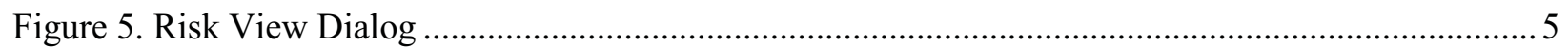


This page intentionally left blank. 


\section{ACRONYMS}

CD compact disk

DDN Design Data Need

NGNP Next Generation Nuclear Plant

PIRT Phenomena Identification and Ranking Table

$\mathrm{R} \& \mathrm{D} \quad$ research and development

RMS Risk Management System

PASSC plant, area, system, subsystem, and component 
This page intentionally left blank. 


\section{NGNP Risk Management Database: A Model for Managing Risk}

\section{System Objective}

To facilitate the implementation of the Risk Management Plan, the Next Generation Nuclear Plant (NGNP) Project has developed and employed an analytical software tool called the NGNP Risk Management System (RMS). A relational database developed in Microsoft ${ }^{\circ}$ Access, this tool provides conventional database utility including data maintenance, archiving, configuration control, and query ability. Additionally, the RMS provides a number of unique capabilities specifically designed to facilitate the development and execution of activities outlined in the Risk Management Plan. Specifically, the RMS provides the capability to establish the risk baseline; document and analyze the risk reduction plan; track the current risk reduction status; organize risks by reference configuration plant, area, system, subsystem, and component (PASSC); and increase the risk knowledge level of NGNP decision making.

\section{System Functions}

To accomplish this objective, the system employs a Hierarchy Viewer that provides a rollup/drilldown analysis capability and summarizes quantitative risk scores at various levels of granularity, as shown in Figure 1. The risk scores can be displayed for either the baseline, current status, or the final projected risk. Risks can be rolled up by average or worse case for a selected design configuration. The tool's Hierarch Viewer also allows the visualization and analyses of the complex relationships between various NGNP data entities (e.g., Critical PASSCs, Risks, Risk Mitigation Tasks, Design Data Needs [DDNs], and Phenomena Identification and Ranking Table [PIRTs]).

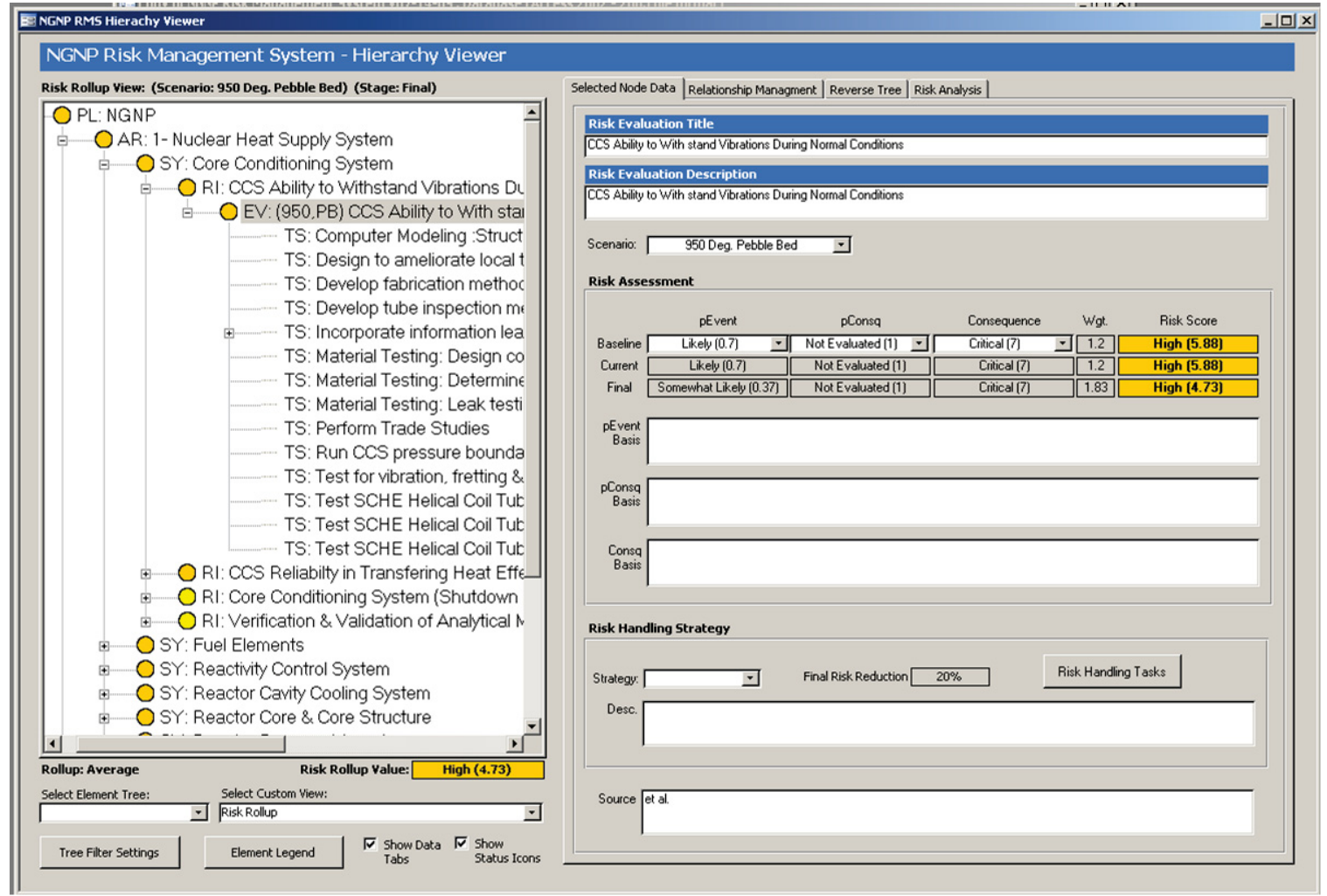

Figure 1. RMS Hierarchy Viewer 
The RMS provides the capability to outline and status a risk handling strategy for each identified risk. Risk reduction tasks are assigned to each risk item and the magnitude of risk reduction estimated for each associated task can be specified, as shown in Figure 2. The status of the risk handling strategy is primarily based on the percent completion of risk reduction tasks. The status of the strategy can also be seen graphically in a Risk Waterfall chart that displays the actual/current risk reduction versus the planned risk reduction over time, as shown in Figure 3.

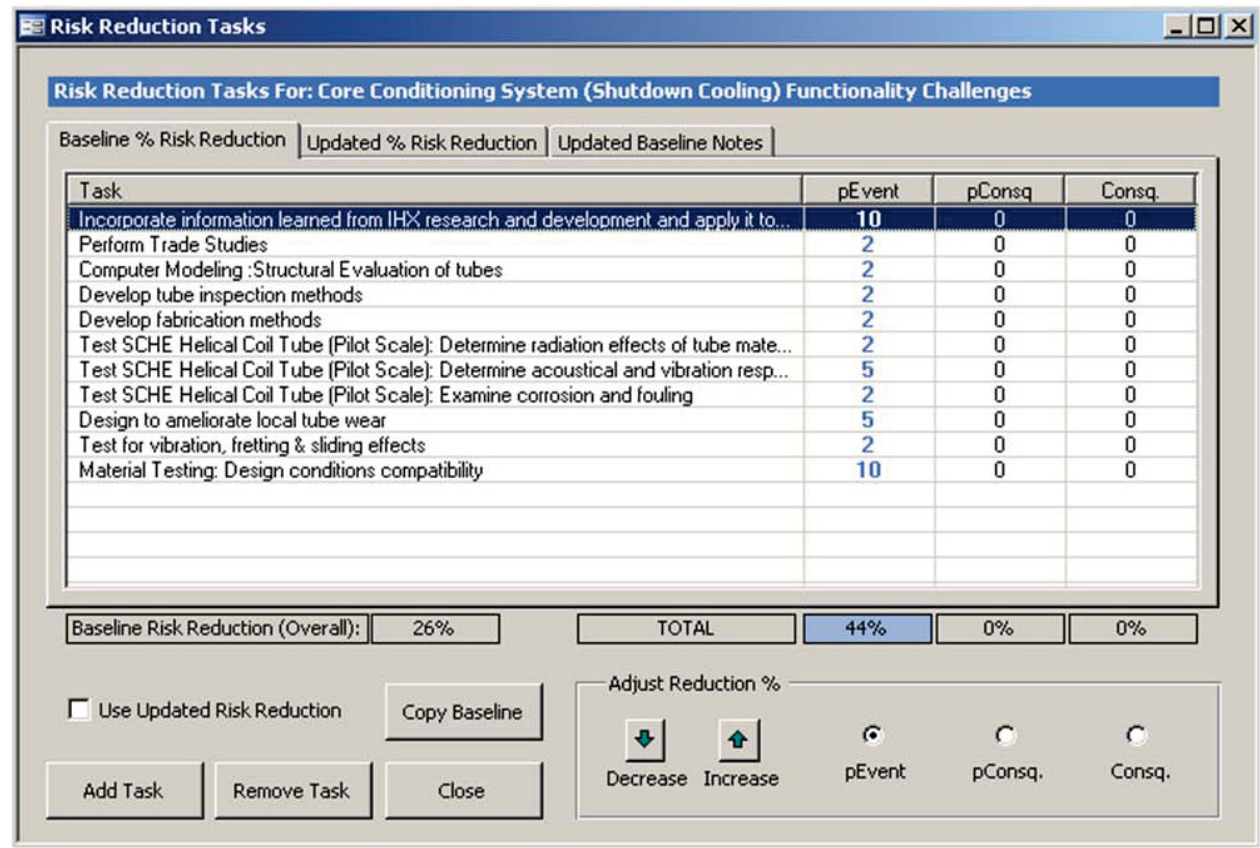

Figure 2. RMP Risk Reduction Tasks

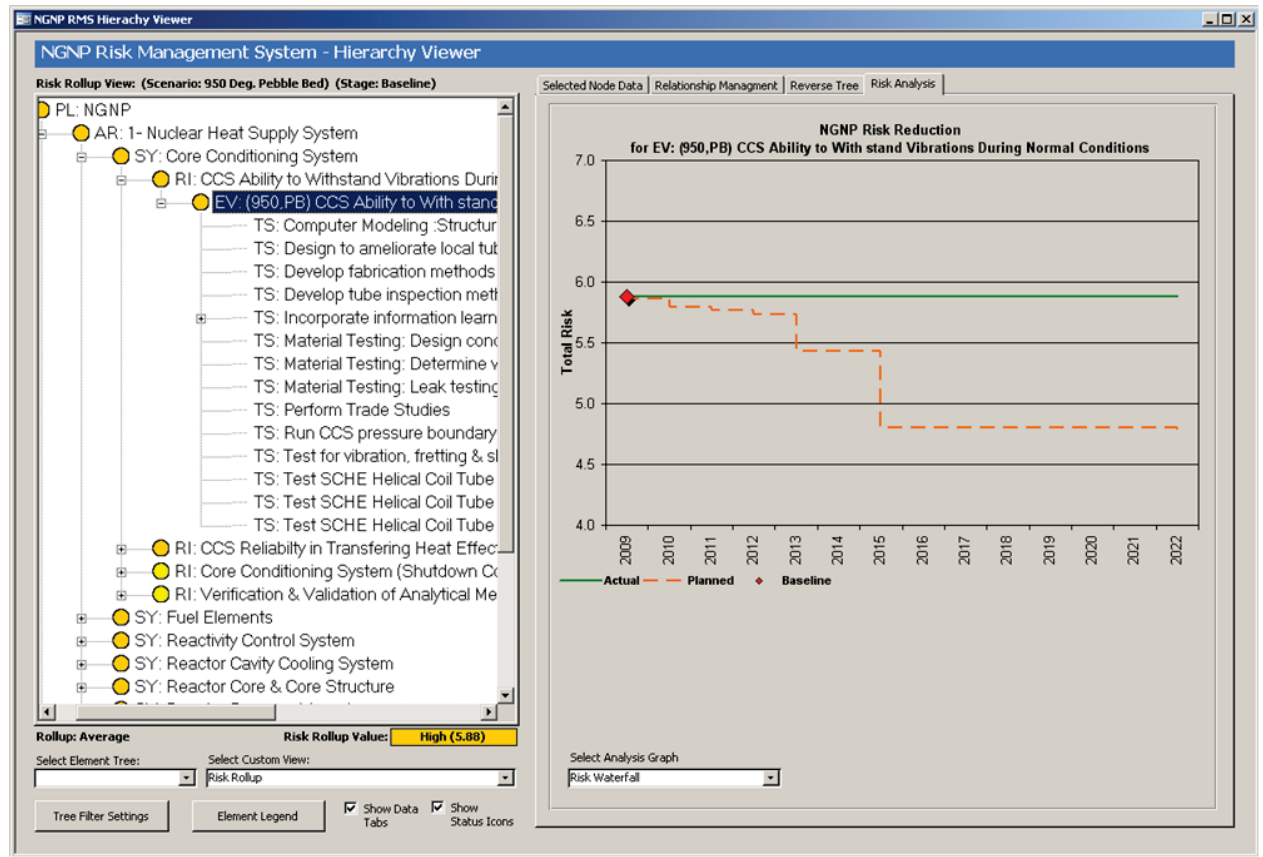

Figure 3. RMS Hierarchy Viewer 
For tasks that provide a reduction in risk for more than one risk item, the tool provides the ability to summarize its contribution across the entire NGNP risk plan. This capability makes it possible to rank order tasks by the magnitude of risk reduction provided for the entire project. This rank ordering of task then provides valuable input into NGNP project planning and prioritization.

Additional RMS functionality includes the ability to analyze and track relational mapping between project risks and PIRTs, risk reduction tasks and DDNs thus facilitating gap identification in planning research and development $(\mathrm{R} \& \mathrm{D})$ activities.

\section{Execution Instructions}

\subsection{Introduction}

This section provides brief instruction on executing some of the key features of the NGNP RMS) The guide is not comprehensive in nature and assumes that users have a certain level of computer skill/expertise in addition to some minimal subject matter expertise in NGNP Risk Management methodology and techniques. The user is referred to the NGNP Risk Management Plan for details regarding NGNP Risk Management.

\subsection{Quick Start}

This section outlines some simple steps to gain access to primary RMS data views. Subsequent sections will provide more detailed instruction.

- Copy the program file (NGNP Risk Management System V1.mde) directly to your computer.

- Start the program by double clicking the file from Windows File Explorer.

- Select "Browse/Edit Relationship Mappings" from the Main Menu.

- Then select “"“Hierarchy Viewer” from the menu.

- Select "Risk Rollup" from the "Select Custom View" drop down menu at the bottom of the screen.

- Select desired parameters from the "Risk View Dialog", and then click "Show View".

- Browse the Hierarchy Tree to view data.

\subsection{System Requirements}

The RMS is based on a relational database developed in Microsoft ${ }^{\circledR}$ Access 2003. As such, any computer properly configured to run Microsoft Access ${ }^{\circledR} 2003$ or higher is adequate to run the RMS. However, be aware that a number of key RMS functions are calculation intensive and older model computers may observe degradation in tool performance.

\subsection{Program Execution}

To start the RMS, copy the program file (NGNP Risk Management System V1.mde) directly to your computer. (The file will not properly execute from a compact disk [CD] or Network Drive.) Then execute the file by either double-clicking the file from Windows File Explorer or by first starting MS Access and then Opening the file directly using MS Access File-Open menu commands. Depending on the version of 
Access the user may need to grant permissions to run macros and other key program features as the program initiates.

\subsection{Key Features}

\subsubsection{Hierarchy Viewer}

The primary user interface in RMS is the Hierarchy Viewer shown in Figure 4. To access the viewer first select "Browse/Edit Relationship Mappings" from the RMS Main Menu, then select "Hierarchy Viewer" from the menu. A key feature of the Viewer is the Hierarchy Window (see Figure 4, Item 3) which provides a graphical representation of RMS data elements and their relationships in a tree view. By expanding the tree the user can drill into various levels of detail. Clicking on any node of the tree provides further detail for that node in the Selected Node Data Tab (see Figure 4, Item 2).

The Hierarchy Viewer provides a variety of ways to view NGNP risk management data in Custom Views as described in Section 3.5.2 below.

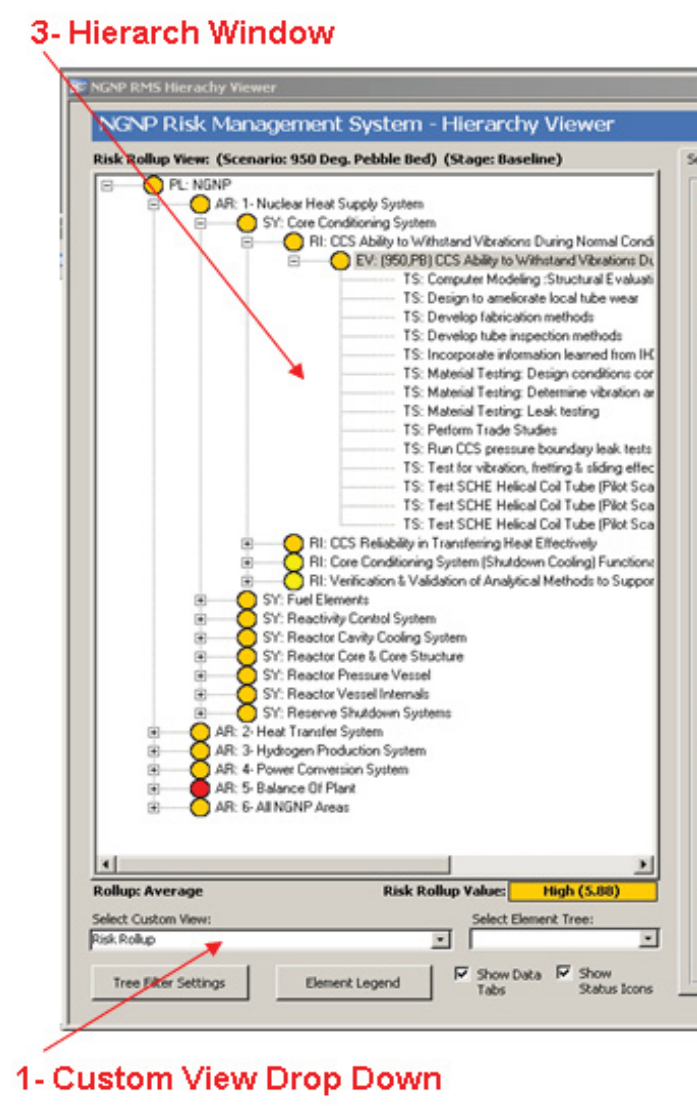

2- Data Tabs

Figure 4. RMS Hierarchy Viewer

\subsubsection{Custom Views}

Custom Views provide graphical visualizations of RMS data each tailored for a specific data perspective. To initiate a Custom View select a view from the Custom View drop down menu shown in Figure 4. There are currently five different Custom Views: 
- $\quad$ Risk Rollup - The risk rollup view is the key RMS data perspective. Selecting this view will first display the Risk View Dialog window (see Figure 5). From this window the user can designate various filters and data view parameters including the Scenario to be viewed, the Risk Values that will be displayed in the Hierarchy Tree and the way the values are rolled up or summarized in the tree from level to level. Once the parameters are chosen click show view to display data in the Viewer. The Dialog window will remain open to allow the user to change parameters as needed. Close the Dialog if not longer required. This view allows the visualization and analyses of the complex relationships between various NGNP data entities (e.g., Critical PASSCs, Risks, Risk Mitigation Tasks, DDNs, and PIRTS). Icons represent a summarization of risk scores at various levels in the Hierarchy. Clicking on/expanding an Evaluation node displays the Risk Handling Tasks, Baseline Risks Scores, Current Risk Scores, Final Risks Scores, and the bases for the scoring. Clicking the Risk Handling Tasks button (see Figure 4, Item 4) shows the percentage Risk Reduction assigned to each task.

- Risk Items (Grouped By Level) - This view groups all applicable risks into Risk Levels and provides a summary count of the risks in each grouping. As with the Risk Rollup the user can browse the associated data by clicking/expanding nodes in the Hierarchy Tree.

- $\quad$ Risk Items (Sorted) - This view displays all applicable risks in the Hierarchy Tree sorted alphabetically. As with the Risk Rollup the user can browse the associated data by clicking/expanding nodes in the Hierarchy Tree.

- Design Data Need (DDN) - This view displays DDNs in the Hierarchy Tree as mapped to associated Risk Handling Tasks. As with the Risk Rollup the user can browse the associated data by clicking/expanding nodes in the Hierarchy Tree.

- $\quad$ Phenomena ID \& Ranking Table (PIRT) - This view displays PIRTs in the Hierarchy Tree as mapped to associated NGNP Risks. As with the Risk Rollup the user can browse the associated data by clicking/expanding nodes in the Hierarchy Tree.

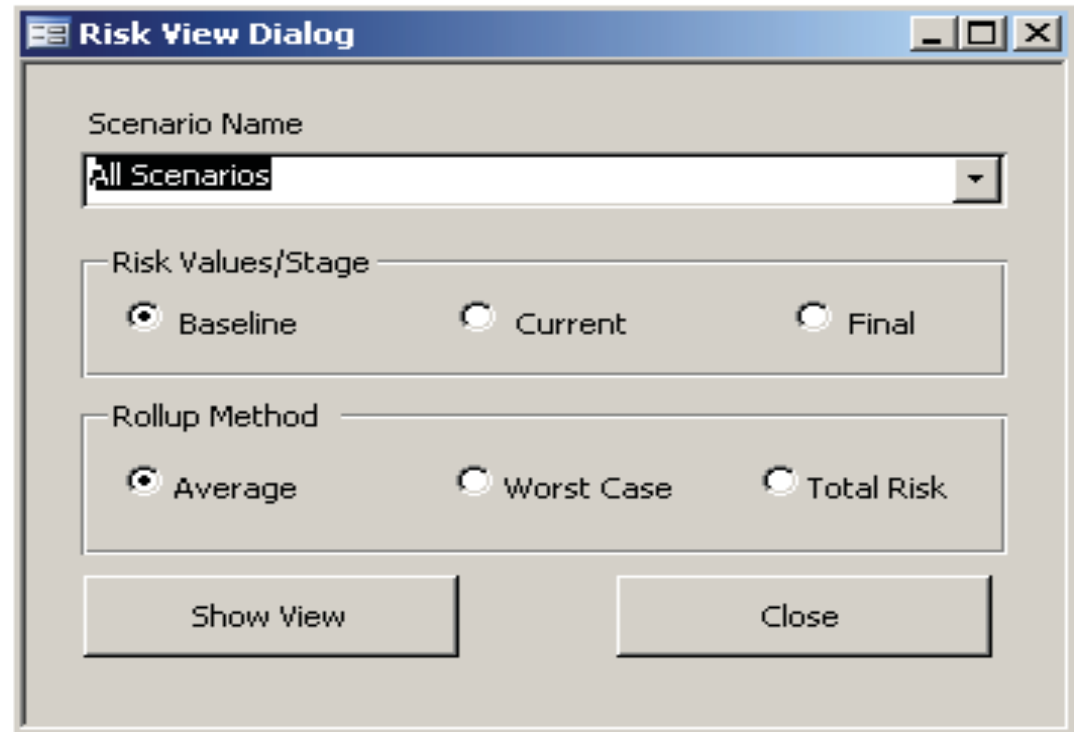

Figure 5. Risk View Dialog 


\subsubsection{Reverse Tree}

The Reverse Tree provides functionality to view crosscutting data relationships for a particular node. For example, a task may provide risk reduction for a number of different risks while the Hierarchy Window may only display one. To explore multiple data mappings for a particular node select the Reverse Tree tab (see Figure 4, Item 2), then select a node in the Hierarchy Window (see Figure 4, Item 3). The reverse tree will display in the tab. The reverse tree can then be expanded up to the root nodes.

\subsubsection{Risk Waterfall Graph}

The status of the risk reduction strategy can seen graphically in a Risk Waterfall chart that displays the actual/current risk reduction versus the planned risk reduction over time. To display the Risk Waterfall chart, first generate the Risk Rollup View (if not already generated), then select the Risk Analysis tab (see Figure 4, Item 2), then select the Risk Waterfall item from the Select Analysis Graph drop down list. The Risk Waterfall chart is designed to be interactive with the Risk Rollup View in the Hierarchy Window. Selecting various node items in the tree will change the basis for the chart. Clicking on the top level NGNP node for example will provide a summary risk reduction chart for the entire NGNP Plant. Similarly, selecting an NGNP Area or NGNP System will provide a risk reduction summary for the selected item.

\subsubsection{Reports}

There are a limited number of reports available in the tool. To access these reports select Reports from the Main Menu, then select Risk Management Reports.

\subsection{Contact Information}

For questions or comments please feel free to contact:

John Collins

Email: John.Collins@inl.gov

Phone: 208-526-3372

NOTE: The native file for the NGNP Risk Management Database will be held at the NGNP Document and Records Storage Center. 